IZA DP No. 5678

Fixed Effects and Variance Components Estimation in Three-Level Meta-Analysis

Spyros Konstantopoulos

April 2011 


\title{
Fixed Effects and Variance Components Estimation in Three-Level Meta-Analysis
}

\author{
Spyros Konstantopoulos \\ Michigan State University \\ and IZA
}

\author{
Discussion Paper No. 5678 \\ April 2011 \\ IZA \\ P.O. Box 7240 \\ 53072 Bonn \\ Germany \\ Phone: +49-228-3894-0 \\ Fax: +49-228-3894-180 \\ E-mail: iza@iza.org
}

\begin{abstract}
Any opinions expressed here are those of the author(s) and not those of IZA. Research published in this series may include views on policy, but the institute itself takes no institutional policy positions.

The Institute for the Study of Labor (IZA) in Bonn is a local and virtual international research center and a place of communication between science, politics and business. IZA is an independent nonprofit organization supported by Deutsche Post Foundation. The center is associated with the University of Bonn and offers a stimulating research environment through its international network, workshops and conferences, data service, project support, research visits and doctoral program. IZA engages in (i) original and internationally competitive research in all fields of labor economics, (ii) development of policy concepts, and (iii) dissemination of research results and concepts to the interested public.
\end{abstract}

IZA Discussion Papers often represent preliminary work and are circulated to encourage discussion. Citation of such a paper should account for its provisional character. A revised version may be available directly from the author. 
IZA Discussion Paper No. 5678

April 2011

\section{ABSTRACT \\ Fixed Effects and Variance Components Estimation in Three-Level Meta-Analysis}

Meta-analytic methods have been widely applied to education, medicine, and the social sciences. Much of meta-analytic data are hierarchically structured since effect size estimates are nested within studies, and in turn studies can be nested within level-3 units such as laboratories or investigators, and so forth. Thus, multilevel models are a natural framework for analyzing meta-analytic data. This paper discusses the application of a Fisher scoring method in two- and three-level meta-analysis that takes into account random variation at the second and at the third levels. The usefulness of the model is demonstrated using data that provide information about school calendar types. SAS proc mixed and HLM can be used to compute the estimates of fixed effects and variance components.

JEL Classification: $\quad$ CO0

Keywords: multilevel models, meta-analysis, random effects

Corresponding author:

Spyros Konstantopoulos

College of Education

Michigan State University

Erickson Hall

East Lansing, MI 48824

USA

E-mail: spyros@msu.edu

\footnotetext{
* I thank Larry Hedges for his valuable comments. Thanks are also due to Harris Cooper and Jeff Valentine for providing the data.
} 
The sheer volume of research related to various topics of scientific interest poses the question of how to organize and summarize findings in order to identify and use what is known as well as focus research on promising areas (Garvey \& Griffith, 1971). This need for accumulating research evidence has led to the development of systematic methods for quantitative synthesis of research (Cooper, Hedges, \& Valentine, 2009). Currently, the use of quantitative methods to summarize results from various empirical studies that test the same hypothesis is widespread in education, psychology, medicine, and social science research. Metaanalysis is a statistical method used to combine evidence from different primary research studies that test comparable hypotheses for the purposes of summarizing evidence and drawing general conclusions (Cooper et al., 2009; Glass, 1976; Hedges \& Olkin, 1985; Lipsey \& Wilson, 2001). Meta-analytic methods involve first describing the results of individual studies via numerical indexes that are commonly called effect size estimates (e.g., correlation coefficient, standardized mean difference, odds ratio) and second combining these estimates across studies to obtain a summary statistic such as a mean (e.g., a standardized mean difference or an average association).

Meta-analytic data are naturally hierarchically structured. For instance, effect sizes are nested within studies, which can be nested within investigators, and so forth. Hence, multilevel models can provide a useful framework for analyzing meta-analytic data and take into account variation in all levels of the hierarchy. Multilevel models have been used extensively over the last 20 years (Goldstein, 1987; Longford, 1993; Raudenbush \& Bryk, 2002; Snijders \& Bosker, 1999) and their applications to meta-analytic data with a two-level structure have been demonstrated in the literature (DerSimonian \& Laird, 1986; Goldstein et al., 2000; Hedges \& Olkin, 1985; Hox \& DeLeeuw, 2003; Raudenbush \& Bryk, 2002). In this paper I discuss the 
application of a Fisher scoring algorithm to univariate two- and three-level meta-analysis. This algorithm computes the fixed effects and the variance components of the random effects within a maximum likelihood framework (Konstantopoulos, 2003; Longford 1987, 1993). I use metaanalytic data with a two- and three-level nested structure that include information about the effects of modified school calendars on student achievement to show the usefulness of the models.

\section{Statistical Models for Meta-Analysis}

Two statistical models have been developed for inference about effect size data from a collection of studies: the random or mixed and the fixed effects models for meta-analysis (Hedges \& Vevea, 1998; Konstantopoulos, 2007). Both models are appropriate for computing estimates in meta-analysis and the choice of model depends on the data structure and the assumptions about the statistical model. Fixed effects models treat an effect size parameter as unknown but fixed and assume that the between-study heterogeneity of the study-specific estimates is virtually zero (Hedges, 1982). Random effects models however, treat the effect size parameters as if they were a random sample from a population of effect size parameters (DerSimonian \& Laird, 1986; Hedges, 1983; Raudenbush \& Bryk, 2002). The random effects model introduces heterogeneity among the effect size parameters that is estimated by the between-study variance (Hedges \& Vevea, 1998).

The simplest random effects model follows typically a two-level structure and introduces a source of variation at the second level, by taking into account the between-study variance of the study-specific estimates (Hedges \& Olkin, 1985; Raudenbush \& Bryk, 2002). Random effects models with a two-level structure have already been developed employing the method of moments or maximum likelihood methods (Goldstein et al., 2000; Hedges \& Vevea, 1998; 
Raudenbush \& Bryk, 2002). Two-level models for multivariate meta-analysis that take into account the dependency in the data have also been discussed in the literature (Berkey, Anderson, \& Hoaglin, 1996; Gleser \& Olkin, 2009). In addition, a recent study discussed the dependency of effect sizes within a study using both a random effects and a hierarchical Bayes approach (Stevens \& Taylor, 2009). The key underlying principle in two-level models is that the effect size parameter is not fixed; instead it has its own distribution and is treated as a random variable at the second level. The between studies model introduces the inconsistency or heterogeneity in study effects across the sample of studies.

Although a two-level model captures the random variation between studies, it does not account for higher levels of nesting in meta-analytic data. Sometimes, however, higher-level nesting takes place in meta-analytic data as with other kinds of data. For example, some researchers have shown empirically the importance of modeling achievement data using threelevel models that take into account nesting effects at the second level (e.g., classroom) and at the third level (e.g., school) (Bryk \& Raudenbush, 1988; Nye, Konstantopoulos, \& Hedges, 2004). Similarly, workers can be nested within departments and firms, and patients can be nested within clinics and hospitals. Meta-analytic data could also have a three-level structure. For example, effect sizes are nested within studies, and studies are nested within level-3 units such as laboratories or investigators. Third level units could also be firms, hospitals, neighborhoods, cities, etc, and the choice of the third level unit depends on the nature and structure of the data. The idea is that studies conducted by the same investigator for example, will likely produce estimates that are correlated and this dependency needs to be taken into account in the analysis. In other words, studies are clustered into investigators. In this case, random variation is evident 
both at the second and at the third levels, since study- and investigator-specific effects can be modeled as random effects at the second and third levels respectively.

In a three-level model the random variation is divided into two parts: the between-study within-level-3 unit variation and the between-level-3 unit variation. This decomposition of the variance is important and informative since it indicates where most of the random variation lies, within or between level-3 units. If the third-level variance is considerable, then it should be included in the estimation process instead of being omitted or collapsed at the second level. In the three-level model level-3 unit estimates form a distribution of effects with a variance that shows differences in effect sizes between level-3 units.

It is difficult to know exactly the optimal number of units that are needed to compute variance components in two- or three-level models. As in the two-level case three-level models involve computations of variances of random effects and in principle larger sample sizes at the third level are preferred because more information is used in the estimation. For example, when there are 10 or more level-3 units a three-level model may be warranted. In contrast, when the number of level-3 units is very small (e.g., 2 to 4) perhaps a two-level model should be used and the effects of the third level units could be modeled as fixed effects (via dummy indicators) at the second level. In addition, ideally, each level-3 unit should include multiple studies. Note that the question about the optimal number of units needed to compute variances of random effects is not inherent to meta-analysis and applies to any two- or three-level model.

In this study I discuss multilevel models for meta-analysis and focus on three-level models. I use an iterative computational algorithm called Fisher scoring to obtain maximum likelihood estimates for two- and three-level models. This method updates the estimates of fixed 
effects and variance components in each iteration using the expected information matrix (see Longford, 1993).

\section{Example}

To illustrate the usefulness of the second and third level models let's consider an example about modified school calendars. The data include studies on schools that modified their calendars without extending the length of the school year (see Cooper, Valentine, Charlton, \& Melson, 2003). The sample of studies used here is somewhat different than that used in the Cooper et al. study, but it suffices for the purposes of the exercise. Overall, 56 studies were included in the sample. First, consider the two-level case, where the first level involves a withinstudy model and the second level a between-study model. Each study provided information that allowed Cooper et al. to construct effect sizes and their standard errors. In addition, there was information about the year of the study which can be modeled as a study-specific predictor. Now, consider the three-level case. The 56 studies were conducted by school districts and thus, studies were nested within districts. Overall, there were 11 school districts (nearly 5 studies per district). In this case, the first level involves a within-study model, the second level a betweenstudy within-district model, and the third level a between-district model. The year of study can be included in the model as a predictor in the third level since it is a district specific variable. The data are summarized in Table 1. Of course, one could imagine a similar structure for health data. For instance, suppose that neighborhoods conduct studies about treatment effects for patients in hospitals in these neighborhoods. 


\section{Two-Level Meta-Analysis}

First I illustrate the method in the simplest case that involves two levels. For simplicity suppose that there is only one outcome in each study and that one effect size estimate is computed in each study (i.e., univariate case). Suppose now that there are $k$ effect size population parameters $\vartheta_{1}, \ldots, \vartheta_{k}$ and therefore $k$ corresponding independent effect size estimates $T_{1}, \ldots, T_{k}$ with known sampling variances $v_{1}, \ldots, v_{k}$. In our example, the $T_{i}$ 's are given in column three of Table 1 , the $v_{i}$ 's are given in column four, and the total number of studies $k$ is 56 . We assume that these effect size estimates $T_{i}$ are independently and normally distributed about $\vartheta_{i}$ with a mean of $\vartheta_{i}$ and variance $v_{i}$. The variances (the $v_{i}$ 's) are unknown, but they are estimated by a consistent estimator and therefore they are assumed to be known (see Table 1). The first level of the hierarchy the within-study model is

$$
T_{i}=\vartheta_{i}+\varepsilon_{i}
$$

where the error term is normally distributed with a mean of zero and a variance $v_{i}$. At the second level of the hierarchy the between-study model the population parameter varies around an overall mean, namely

$$
\vartheta_{i}=\beta_{0}+\eta_{i}
$$

where $\eta_{i}$ is a study-specific random effect that is normally distributed with a mean of zero and variance $\tau$ ( $\tau>0)$. In a single level notation the model is written as

$$
T_{i}=\beta_{0}+\eta_{i}+\varepsilon_{i}
$$

The second level can also include $p$ predictors (e.g., study characteristics such as the year of the study) namely 


$$
\vartheta_{i}=\beta_{0}+\beta_{1} X_{1 i}+\ldots+\beta_{p} X_{p i}+\eta_{i}
$$

and in this regression model the residual variance of the random effect $\eta$ is $\tau_{R}$. In our example, one predictor is year of study and is reported in the last column of Table 1. In the Cooper at al. study other study level predictors are also reported such as whether the study was conducted by an internal of external evaluator.

For the within-study model we assume that the variances of the stochastic errors are different for each study (i.e., heterogeneity of the sampling error), while for the between-study model we assume that the random effects are distributed identically (i.e., homogeneity of random effects). The sampling error variances in meta-analytic data cannot be expected to be identical across studies since they typically depend on the sample size of each study and hence, the heterogeneity assumption seems reasonable. The units at each level are independently distributed and thus the error terms $\varepsilon_{i}$ and $\eta_{i}$ at the first and the second level respectively are uncorrelated, that is $\operatorname{cov}\left(\varepsilon_{i}, \varepsilon_{j}\right)=0$, and $\operatorname{cov}\left(\eta_{i}, \eta_{j}\right)=0$. In a single level equation the two-level model with second level predictors is

$$
T_{i}=\beta_{0}+\beta_{1} X_{1 i}+\ldots+\beta_{p} X_{p i}+\eta_{i}+\varepsilon_{i} .
$$

The effect sizes $T_{i} s$ are normally distributed with a mean $\beta_{0}+\sum_{j=1}^{p} \beta_{j} X_{i j}$, and a variance $v_{i}+\tau_{R}$, and when there are no predictors in the model the between-study variance is $\tau$.

\section{Estimation}

I compute estimates of the fixed effects, the regression coefficients, and the variance components of the random effects at the second level using maximum likelihood estimation. I 
used a Fisher scoring algorithm to compute the maximum likelihood estimates following Longford (1987, 1993). For simplicity, I discuss the simplest two-level case with no predictors at the second level. The advantage of the simplest case is that the estimates have simple algebraic expressions. Even the inclusion of one predictor in the model complicates the expressions considerably. The more general case that can include predictors is expressed in matrix notation and is illustrated in the appendix. The idea is to maximize a log-likelihood function in order to estimate the fixed effects and the variance components of the random effects (see appendix). The Fisher scoring algorithm involves the computation of the first and second order derivatives of the parameter estimates, the fixed effects and variance components.

In the simplest case, where there are no study-level predictors included in the model, the objective is to compute one overall mean, the intercept, and the second level random effects variance component $\tau$. For example, the fixed effects estimate or overall mean in this case is a product of sums as shown in the Fisher scoring equation

$$
\left\{\sum_{i=1}^{k}\left(v_{i}+\tau\right)^{-1}\right\}^{-1} \sum_{i=1}^{k} T_{i}\left(v_{i}+\tau\right)^{-1}
$$

where $\sum_{i=1}^{k} T_{i}\left(v_{i}+\tau\right)^{-1}$ is the scoring function and $\sum_{i=1}^{k}\left(v_{i}+\tau\right)^{-1}$ is the expected information function. The Fisher scoring equation when predictors are included in the model is given in the appendix (A-3). Similarly, the between-study variance component at the second level is updated as

$$
\tau=\tau_{0}-2\left\{\sum_{i=1}^{k}\left(v_{i}+\tau\right)^{-2}\right\}^{-1} \frac{1}{2}\left\{\sum_{i=1}^{k}\left(v_{i}+\tau\right)^{-1}-\sum_{i=1}^{k} e_{i}^{2}\left(v_{i}+\tau\right)^{-2}\right\}
$$


where $e$ is a residual defined in the appendix, $\tau_{0}$ is the initial estimate of the second level variance, $\quad 1 / 2 \sum_{i=1}^{k}\left(v_{i}+\tau\right)^{-2} \quad$ is the expected information function and $-1 / 2\left\{\sum_{i=1}^{k}\left(v_{i}+\tau\right)^{-1}-\sum_{i=1}^{k} e_{i}^{2}\left(v_{i}+\tau\right)^{-2}\right\}$ is the scoring function. The variance of the fixed effect estimate is given by $\left\{\sum_{i=1}^{k}\left(v_{i}+\tau\right)^{-1}\right\}^{-1}$ and the variance of the variance component is given by $2\left\{\sum_{i=1}^{k}\left(v_{i}+\tau\right)^{-2}\right\}^{-1}$. Convergence is achieved when the log-likelihood remains unchanged for several decimal places.

\section{Three-Level Meta-Analytic Model}

The computations in the three-level model are more complicated mainly because of the estimation of the variance components at the third level. Again, suppose that there is only one outcome and one effect size estimate per study. For simplicity let's consider first the simplest case where no predictors are included at levels 2 and 3. The computation involves an overall mean estimate and two variance component estimates at levels 2 and 3. Simple algebraic expressions are not always possible in the three-level model. The model for the first level of the hierarchy (the within-study model) for effect size estimate $T_{i}$ is identical to equation (1).

In the second level of the hierarchy (the between-study within-level-3-unit model) the unknown effect-size parameter $\vartheta$ varies around a level-3 unit g mean, namely

$$
\vartheta_{i g}=\beta_{0 g}+\eta_{i g}
$$


where $g=1, \ldots, m$ represents the level-3 units (e.g., school district). Finally, at the third level the level-3 unit means vary around an overall mean $\gamma_{00}$, namely

$$
\beta_{0 g}=\gamma_{00}+v_{0 g},
$$

where $v_{0 g}$ is a level-3 unit specific random effect that is normally distributed with a mean of zero and variance $\omega(\omega>0)$. In our example, $\omega$ is the between-district variance. In a single level notation the model is written as

$$
T_{i g}=\gamma_{00}+v_{0 g}+\eta_{i g}+\varepsilon_{i g}
$$

Now when $p$ predictors are include at the second level the model is

$$
\vartheta_{i g}=\beta_{0 g}+\beta_{1 g} X_{1 i g}+\ldots+\beta_{p g} X_{p i g}+\eta_{i g}
$$

where $X_{1 i g}, \ldots, X_{p i g}$ are study-specific predictors (e.g., year of study), $\beta_{0 g}, \beta_{1 g}, \ldots, \beta_{p g}$ are unknown regression coefficients that need to be estimated, and $\eta_{i g}$ is a level-2 random effect or residual. The residuals at the second level are independently, identically, and normally distributed with a mean of zero and a residual variance $\tau_{R}$. The third level model for the level-3 unit mean (or intercept) $\beta_{0 g}$ when $q$ level-3 predictors are included in the model is

$$
\beta_{0 g}=\gamma_{00}+\gamma_{01} W_{1 g}+\ldots+\gamma_{0 q} W_{q g}+v_{0 g},
$$

where subscript $\mathrm{R}$ indicates residual variance, $W_{1 g}, \ldots, W_{q g}$ are level-3 unit-specific predictors (e.g., school district characteristics), $\gamma_{00}, \gamma_{01}, \ldots, \gamma_{0 q}$ are unknown regression coefficients that need to be estimated, and $v_{0 g}$ is a level-3 random effect or residual that is normally distributed with a mean of zero and residual variance $\omega_{R}$. In this model the year of study can be included in the third level as a school district predictor, and $\omega_{R}$ is the residual between-district variance. 
The model illustrated in equation (14) is also used to model the level-2 slopes in equation (13). The study-specific characteristics are modeled either as fixed or random effects at the third level. Level-3 predictors can be used to model level-2 slopes as shown in equation (14). In our example, a study characteristic is whether the study was conducted by an internal or an external evaluator (see Cooper et al., 2003). The effect of this variable could vary by school district (the third level unit) perhaps because the internal evaluator effects are more pronounced in some districts, but weaker in others. As a result, it is possible that the evaluator effect varies by district. The evaluator effect is in this case a random effect and is known as a cross-level interaction between the evaluator effect and school districts. When the variance of this random effect is significant, then there is evidence of interaction.

In another example, suppose that a good number of researchers study differences in achievement between small and regular classes. Also, suppose that each researcher conducted multiple studies on this topic, that is, studies are nested within investigators. One study characteristic of interest is the type of research design the study used, whether for example, the study was a randomized experiment or not. Let's assume that each researcher conducted both experiments and non-experiments. The research design effect may interact with researchers and as a result it may vary across researchers. That is, the research design effect could be smaller for some researchers and larger for others. Study characteristics can also interact with specific level3 unit characteristics and in this case the cross-level interaction is modeled as a fixed effect. In this example, the research design effect may interact with the experience or the training of the researcher in the field.

When $p$ level-3 unit-specific slopes are regressed on predictors at the third level and are treated as random at the third level, these random effects are normally distributed with a mean of 
zero and a variance-covariance matrix $\Omega_{(3) R}$ with $p+1$ residual variance components in the diagonal and covariances among these variances in the off diagonal, and $\boldsymbol{v}_{g}$ is the vector of the $p+1$ level-3 random effects within level-3 unit g. The omega matrix is a $(p+1) \mathrm{x}(p+1)$ symmetric matrix.

The variance of an effect size estimate, $T_{i}$, in the simplest three-level model assuming no predictors at any level is

$$
\operatorname{Var}\left(T_{i}\right)=v_{i}+\tau+\omega
$$

where $\tau$ is the level-2 variance component and $\omega$ is the level-3 variance component. However, when predictors are included at the second level and are treated as random effects at the third level, the variance of an effect size estimate $T_{i}$ is

$$
\operatorname{Var}\left(T_{i}\right)=\mathrm{v}_{i} \tau+{ }_{R}^{2}+\mathbf{z}_{i g}^{T} \boldsymbol{\Omega}_{(3) R} \mathbf{z}_{i g}
$$

where $\mathbf{z}_{i g}$ is a $(p+1) \times 1$ vector assuming $p+1$ level- 2 slopes modeled as random effects at the third level. For example, if the intercept and evaluator effect are modeled as random effects at the third level the vector $\mathbf{z}$ is a $2 \mathrm{x} 1$ vector and the omega matrix if a $2 \times 2$ variance matrix. The random effects at the third level are the level-3 intercepts and slopes. The data are nested in the third level since each level-3 unit (e.g., school district) will include multiple level-two units (e.g., studies).

\section{Estimation}

In the three-level model we also need to maximize the log-likelihood function in order to estimate the fixed effects and the variance components of the random effects (see appendix). The log-likelihood equation is identical to that in the two-level model. However, the design matrix $\mathbf{X}$ 
of the fixed effects or predictors and the design matrix $\mathbf{Z}$ of the random effects are more complicated in the three-level case since more predictors and random effects are introduced in the regression equation (see appendix). In the following discussion I illustrate the estimation for the simplest case that involves an overall fixed effect, the weighted mean, and two variance components, one at the second and one at the third level.

Consider the simplest error structure at the third level, where only level-3 unit intercepts are random at the third level and there are no predictors at any level. Then the design matrix of the random effects for unit $\mathrm{g}$ at the third level is $\mathbf{Z}_{(3, g)}=\mathbf{1}_{\left(n_{g}\right)}$, where $\mathbf{1}_{\left(n_{g}\right)}$ is a vector of ones that has as many elements as the number of studies within unit g and $\mathbf{Z}_{(3, g)} \boldsymbol{\Omega}_{(3)} \mathbf{Z}_{(3, g)}^{T}=\omega \mathbf{J}_{\left(n_{g}\right)}$, where $\mathbf{J}_{\left(n_{g}\right)}=\mathbf{1}_{\left(n_{g}\right)} \mathbf{1}_{\left(n_{g}\right)}^{T}$ is a $n_{g}$ X $n_{g}$ matrix of ones. To illustrate the variance structure suppose that the level-3 unit g has 3 studies; then the structure of the variance-covariance matrix $\mathbf{V}_{(3, g)}$ within a level-3 unit g when predictors are not included at any level is

$$
\mathbf{V}_{(3, g)}=\left[\begin{array}{ccc}
v_{1}+\tau & 0 & 0 \\
0 & v_{2}+\tau & 0 \\
0 & 0 & v_{3}+\tau
\end{array}\right]+\omega\left[\begin{array}{lll}
1 & 1 & 1 \\
1 & 1 & 1 \\
1 & 1 & 1
\end{array}\right]=\left[\begin{array}{lcc}
v_{1}+\tau+\omega & \omega & \omega \\
\omega & v_{2}+\tau+\omega & \omega \\
\omega & \omega & v_{3}+\tau+\omega
\end{array}\right]
$$

The variance structure illustrated above is similar when level-2 and level-3 predictors are included in the model as fixed effects. The only difference is that the level-2 and level-3 variances are now residual variances, $\tau_{R}, \omega_{R}$. When level-2 slopes are treated as random effects at the third level however, the variance structure is more complicated and includes the additional variance components and the design matrixes of these random effects (see appendix).

The computation of the log-likelihood involves the inverse of the variance-covariance matrix $\mathbf{V}$ as well as its determinant. When predictors are not included at any level the between- 
study variance is $\tau$ and the between level-3 unit variance is $\omega$. Now consider a $n_{g} \times n_{g}$ diagonal matrix $\mathbf{D}$, defined as $\mathbf{D}_{\left(n_{g}\right)}=\operatorname{diag}\left(v_{i}+\tau, i=1, \ldots, n_{g}\right)$, and $n_{g}$ represents the number of studies within a third level unit $g$. Then, the inverse of the variance-covariance matrix for a third level unit $g$ is

$$
\mathbf{V}_{(3, g)}^{-1}=\mathbf{D}_{\left(n_{g}\right)}^{-1}-\left(\omega^{-1}+\sum_{i=1}^{n_{g}}\left(v_{i}+\tau\right)^{-1}\right)^{-1} \mathbf{D}_{\left(n_{g}\right)}^{-1} \mathbf{J}_{\left(n_{g}\right)} \mathbf{D}_{\left(n_{g}\right)}^{-1} .
$$

The diagonal elements of matrix $\mathbf{V}_{(3, g)}^{-1}$ are

$$
\left(v_{i}+\tau\right)^{-1}\left\{1-\left(v_{i}+\tau\right)^{-1}\left(\omega^{-1}+\sum_{i=1}^{n_{g}}\left(v_{i}+\tau\right)^{-1}\right)^{-1}\right\}
$$

and the non-diagonal elements of matrix $\mathbf{V}_{(3, g)}^{-1}$ are

$$
-\left(v_{i}+\tau\right)^{-1}\left(v_{j}+\tau\right)^{-1}\left(\omega^{-1}+\sum_{i=1}^{n_{g}}\left(v_{i}+\tau\right)^{-1}\right)^{-1}
$$

The determinants of matrix $\quad \mathbf{V} \quad$ are $\quad\left|\mathbf{V}_{(2, g)}\right|=\prod_{i=1}^{n_{g}}\left(v_{i}+\tau\right), \quad\left|\boldsymbol{\Omega}_{(3) R}\right|=\omega$,

$$
\begin{gathered}
\left|\boldsymbol{\Omega}_{(3) R}^{-1}+\mathbf{Z}_{(3, g)}^{T} \mathbf{V}_{(2, g)}^{-1} \mathbf{Z}_{(3, g)}\right|=\omega^{-1}+\sum_{i=1}^{n_{g}}\left(v_{i}+\tau\right)^{-1} \text { and as a result } \\
\left|\mathbf{V}_{(3, g)}\right|=\prod_{i=1}^{n_{g}}\left(v_{i}+\tau\right)\left(1+\omega \sum_{i=1}^{n_{g}}\left(v_{i}+\tau\right)^{-1}\right) .
\end{gathered}
$$

In the simplest case where we need to compute the overall mean and the second and third level variance components, the matrix $\mathbf{X}$ is a column vector of ones and the overall fixed effect or mean is computed using the following algebraic forms of the expected information and scoring functions, namely 


$$
\begin{aligned}
& \mathbf{X}^{T} \mathbf{V}^{-1} \mathbf{X}=\sum_{g=1}^{m}\left\{\sum_{i=1}^{n_{g}}\left(v_{i}+\tau\right)^{-1}\right\}_{g}- \\
& \sum_{g=1}^{m}\left\{\left(\omega^{-1}+\sum_{i=1}^{n_{g}}\left(v_{i}+\tau\right)^{-1}\right)_{g}^{-1}\left(\sum_{i=1}^{n_{g}}\left(v_{i}+\tau\right)^{-2}+\sum_{i=1}^{n_{g}\left(n_{g}-1\right)}\left(v_{i}+\tau\right)^{-1}\left(v_{j}+\tau\right)^{-1}\right)_{g}\right\},
\end{aligned}
$$

and

$$
\begin{aligned}
& \mathbf{X}^{T} \mathbf{V}^{-1} \mathbf{T}=\sum_{g=1}^{m}\left\{\sum_{i=1}^{n_{g}} T_{i}\left(v_{i}+\tau\right)^{-1}\right\}_{g}- \\
& \sum_{g=1}^{m}\left\{\left(\omega^{-1}+\sum_{i=1}^{n_{g}}\left(v_{i}+\tau\right)^{-1}\right)_{g}^{-1}\left(\sum_{i=1}^{n_{g}} T_{i}\left(v_{i}+\tau\right)^{-2}+\sum_{i=1}^{n_{g}\left(n_{g}-1\right)} T_{i}\left(v_{i}+\tau\right)^{-1}\left(v_{j}+\tau\right)^{-1}\right)_{g}\right\}
\end{aligned}
$$

where $i \neq j, j=1, \ldots, n_{g}$. Equations (19) and (20) produce scalars and using equation (A-3) one can compute the fixed effects estimate which is essentially the product of equations (19) and (20).

The scoring and expected information functions for the second level variance component $\tau$ are

$$
-\frac{1}{2}\left\{\sum_{g=1}^{m} \operatorname{tr}\left\{\mathbf{V}_{(3, g)}^{-1}\right\}_{g}-\sum_{g=1}^{m}\left\{\left(\mathbf{e}_{(3, g)}^{T} \mathbf{V}_{(3, g)}^{-1}\right)\left(\mathbf{V}_{(3, g)}^{-1} \mathbf{e}_{(3, g)}\right)\right\}_{g}\right\}
$$

and

$$
\frac{1}{2}\left\{\sum_{g=1}^{m}\left\{\operatorname{tr}\left\{\mathbf{V}_{(3, g)}^{-1} \mathbf{V}_{(3, g)}^{-1}\right\}\right\}_{g}\right\}
$$

respectively, where $\mathbf{e}$ is a row vector defined in the appendix, and $\operatorname{tr}(\mathbf{A})$ is the trace of matrix $\mathbf{A}$, the sum of diagonal elements of $\mathbf{A}$. The derivative $\frac{\partial \mathbf{V}}{\partial \tau}$ produces an identity matrix that is not 
used in the computation. Equations (21) and (22) produce scalars and the variance of $\tau$ is computed by taking the inverse of equation (22). In the simplest case

$$
\operatorname{tr}\left\{\mathbf{V}_{(3, g)}^{-1}\right\}=\sum_{i=1}^{n_{g}}\left\{\left(v_{i}+\tau\right)^{-1}\left(1-\left(v_{i}+\tau\right)^{-1}\left(\omega^{-1}+\sum_{i=1}^{n_{g}}\left(v_{i}+\tau\right)^{-1}\right)^{-1}\right)\right\} .
$$

The scoring function for the third level variance component matrix $\boldsymbol{\Omega}_{(3) R}$ for each variance or unique covariance is

$$
-\frac{1}{2}\left\{\sum_{g=1}^{m}\left\{\left(\mathbf{Z}_{(3, g)}^{T} \mathbf{V}_{(3, g)}^{-1} \mathbf{Z}_{(3, g)}\right)_{j l}\right\}_{g}-\sum_{g=1}^{m}\left\{\left(\mathbf{Z}_{(3, g)}^{T} \mathbf{V}_{(3, g)}^{-1} \mathbf{e}_{(3, g)}\right)_{j}\left(\mathbf{Z}_{(3, g)}^{T} \mathbf{V}_{(3, g)}^{-1} \mathbf{e}_{(3, g)}\right)_{l}\right\}_{g}\right\}
$$

where $j, l$ indicate the $j$ th row and $l$ th column element of the matrix. The derivative $\frac{\partial \Omega_{(3) R}}{\partial \omega_{R j l}}$

produces an incidence matrix with zeros everywhere and one for element $j, l$ (Longford, 1993).

The diagonal elements (variances) of the expected information matrix for the third-level variance components (variances and lower triangular matrix covariances) are computed as

$$
\frac{1}{2}\left\{\sum_{g=1}^{m}\left\{\left(\mathbf{Z}_{(3, g)}^{T} \mathbf{V}_{(3, g)}^{-1} \mathbf{Z}_{(3, g)}\right)_{j l}\left(\mathbf{Z}_{(3, g)}^{T} \mathbf{V}_{(3, g)}^{-1} \mathbf{Z}_{(3, g)}\right)_{j l}\right\}_{g}\right\},
$$

A similar equation is used to compute the off-diagonal elements, the covariances between the variance and the covariance components, namely

$$
\sum_{g=1}^{m}\left\{\left(\mathbf{Z}_{(3, g)}^{T} \mathbf{V}_{(3, g)}^{-1} \mathbf{Z}_{(3, g)}\right)_{j m}\left(\mathbf{Z}_{(3, g)}^{T} \mathbf{V}_{(3, g)}^{-1} \mathbf{Z}_{(3, g)}\right)_{k l}\right\}_{g}
$$

where $j, m$ and $k, l$ are elements of the matrices.

We assume that the random effects at different levels have a zero covariance, that is, they are not correlated. In the simplest case the derivative $\frac{\partial \mathbf{V}_{(3, g)}}{\partial \omega}=\mathbf{J}_{n_{g}}$ is a $n_{g} \times n_{g}$ matrix of ones and 
hence $\operatorname{tr}\left(\mathbf{V}_{(3, g)}^{-1} \frac{\partial \mathbf{V}_{(3, g)}}{\partial \omega}\right)=\operatorname{tr}\left(\mathbf{V}_{(3, g)}^{-1} \mathbf{J}_{n_{g}}\right)=\sum_{i=1}^{n_{g}^{2}} a_{i}$ is the sum of all elements of $\mathbf{V}_{(3, g)}^{-1}$, and $a_{i}$ represents an element of $\mathbf{V}_{(3, g)}^{-1}$.

Reasonable starting values (indicated by subscript 0) for the fixed effects estimates are estimates produced from ordinary least squares. Following Longford (1993) reasonable starting solutions for $\tau_{R}$ and the diagonal elements of the variance components matrix $\boldsymbol{\Omega}_{(3) R}$ at the third level are functions of the residuals namely

$$
\tau_{(0) R}=\frac{1}{k} \sum_{i=1}^{k} e_{i}^{2}
$$

and

$$
\boldsymbol{\Omega}_{(0) R j}=\frac{1}{m} \frac{\sum_{g=1}^{m}\left(\mathbf{e}_{(3, g)}^{T} \mathbf{Z}_{(3, g)}^{(j)}\right)^{2}}{\sum_{g=1}^{m}\left(\mathbf{Z}_{(3, g)}^{(j)}\right)^{T} \mathbf{Z}_{(3, g)}^{(j)}}
$$

where the superscript $j$ represents the $j$ th diagonal element of the third level variance components matrix $\Omega$.

\section{Data}

In the school calendar example the studies were nested within school districts and thus a third level, the school district, was introduced in the model (Cooper et al., 2003). In the data I analyzed, each school district included at least three studies. The first level involves a withinstudy model, the second level a between-study within-district model, and the third level a between-district model. Criteria for selection were complete data that provided information on effect size estimates and type of calendar. All studies assessed students from grade 1 through 
Three-Level Meta-Analysis

grade 9 and reported achievement differences between students attending schools that follow a year-round calendar and schools that follow the traditional nine-month calendar. The achievement differences were expressed in standard deviation units to ensure all estimates were on the same scale. The data included information on reading achievement, and included 56 studies nested within 11 school districts. Positive effect sizes indicated that students in schools that followed a year-round calendar performed higher on average than students in schools that followed the traditional nine-month calendar. I first ran a two-level and then a three-level model. I also ran two different specifications. The first specification was an unconditional model with no predictors. This model estimates an overall mean as a fixed effect, and the variances at the second and third levels. The second specification added a predictor, the year the study was conducted. The year of study was used at the top level in the two- or the three-level model.

\section{Using Software to Compute Estimates}

To compute the fixed effects and the variance components one can use either the SAS or the HLM software. The procedure proc mixed in SAS is well-suited for two- and three-level univariate meta-analysis. Similarly, the windows version of HLM produces estimates for twoand three-level univariate meta-analysis. SAS proc mixed is a general purpose routine that can be used for fitting random effects models (Singer, 1998). The codes I used to analyze the data with SAS proc mixed are presented in the appendix for unconditional models. Predictors can of course be included in the regression equation. However in order to obtain an intercept that represents an adjusted, by the predictors, average effect size estimate the predictors need to be centered around their grand mean before they are included in the equation. Detailed information about the proc mixed procedure is provided by Littell et al. (1996), Konstantopoulos and Hedges 
(2004), and Singer (1998). Alternatively, one could use the HLM software that is designed especially for fitting multi-level models. The windows version of HLM can be used to fit twoand three-level meta-analysis models (Raudenbush, Bryk, Cheong, \& Congdon, 2004). The code created by HLM for two- and three-level meta-analysis is also reported in the appendix for unconditional models. In HLM the user can choose to grand-mean center predictors in order to obtain a meaningful intercept, the overall effect size adjusted by predictors. The user needs to specify in the estimation settings that the first level variances are known and that the weighting variable is the variance of the effect size. Detailed information about the HLM software is provided by Raudenbush et al. (2004).

\section{Results}

The studies included in the sample were conducted between 1976 and 2000 (see Table 1). Approximately $34 \%$ of the samples were from studies conducted in 1976, while $27 \%$ were from studies conducted in 1997. The remaining 39\% were from studies conducted in 1989 (7\%), 1994 (9\%), 1995 (3.5\%), and 2000 (19.5\%). Approximately 61\% of the samples were obtained from dissertations or theses, while the remaining samples were obtained from journal articles (18\%), school reports (7\%), or studies by the research departments of school districts (14\%). Nearly $44 \%$ of the samples were obtained from studies conducted in large urban areas, another $35 \%$ from studies in small urban or suburban areas, and the remaining $21 \%$ from studies in rural areas. The samples included studies conducted in grades one through nine. All standardized mean differences or effect sizes used in the data do not reflect adjustments for covariates, and thus were unadjusted differences between school calendar and traditional calendar schools. 
Insert Table 1 Here

The effect size estimates ranged from -0.52 to 1.19 with a mean of 0.12 and a standard deviation of 0.33 (see Table 2). Negative effect sizes indicate that students attending traditional (nine-month) calendar schools outperformed their counterparts in year-round schools. In contrast, positive effect sizes point to higher student achievement in year-round schools. The sample sizes ranged from 28 to 4,403 students with a mean of 913 students. About $52 \%$ of the samples were from year-round schools on a nine-week instruction followed by a three-week break schedule, $12.5 \%$ from schools on a twelfth-week instruction followed by a four-week break schedule, and nearly 35.5\% from schools on other types of schedules.

\section{Insert Table 2 Here}

The dataset has an unbalanced structure. There were 11 level-3 units (districts) and within each district the number of studies ranged from 3 to 11 with an average of 5.1. Table 3 reports means and standard deviations for effect size estimates by district. It appears that there is considerable variability within as well as between districts. District 71 had the highest mean and second highest standard deviation, while district 11 had the lowest mean. District 108 had the largest standard deviation and district 86 had the lowest standard deviation.

Insert Table 3 Here 
The results from the two-level meta-analysis are summarized in Table 4. Specifically, Table 4 reports the estimates of the fixed effects and the variance components, their standard errors (SE), and 95\% confidence intervals (CI) ${ }^{1}$ around the estimates. The overall effect size estimate was 0.128 and significant indicating that on average students in schools that follow year-round calendars outperformed their peers in schools that follow traditional calendars. The between-study variance component was 0.088 and significantly different from zero, which indicates that the effect sizes varied across studies. The range of the $95 \%$ CI was 0.059 to 0.146 . When the year of study was included in the model at the study level the effect size estimate was 0.126 and still significant. The variance component estimate did not change and the year of study was not a significant predictor of the effect sizes and did not explain any of the level-2 variance. The estimate of year of study was very close to zero. Now, using the same data the estimate of the overall mean using fixed effects models was nearly one-half as large as the estimate of the mean using a two-level random effects model because of the different structure of the weight matrix. The estimate of year of study was still very close to zero and insignificant. In addition, the standard error of the weighted mean in the two-level random effects model was 50 percent larger than that in the fixed effects model.

Insert Table 4 Here

The three-level analysis estimates are summarized in Table 5 . The structure of Table 5 is the same as the structure in Table 4. The overall effect size estimate is now 0.184 and significant. The overall weighted mean estimate is different than that in the two-level model or the fixed effects model, because a different weight matrix $\mathbf{V}^{-\mathbf{1}}$ is used in the three-level model 
computation. The variance-covariance matrix is block diagonal in the three-level model, while it is a diagonal matrix in the two-level model (and includes the between study-variance $\tau$ ). In the fixed effects model case the weight matrix is also diagonal but includes only the $v_{i}$ 's. A change in the magnitude of the overall mean should be expected whenever random effects are introduced in the model because the variance components of these random effects are included in the weight matrix. For example, using the same data the estimate of the overall weighted mean using fixed effects models was one-half to one-third as large as the estimate of the mean using a three-level random effects model. In addition, the standard errors of the weighted mean and the year of study using fixed effects models were at least one-half as large as those in the three-level model. Similarly, the standard errors of the weighted mean and the year of study in the three-level model were twice as large than those in the two-level case.

The second level variance was 0.033 and was significantly different from zero. The $95 \%$ CI was 0.020 to 0.070 . The third level variance was almost twice as large and also significantly different from zero. The $95 \%$ CI was 0.027 to 0.256 . Hence, most of the random variation was between districts not between studies within districts. The advantage of the third level model is that it provided a more accurate picture of the dependencies in the data through the variance decomposition. When the level-3 variance is significant and non trivial in magnitude it should be included in the computation of the regression estimates and their standard errors. In the two-level model all random variation was assumed to be between-study variation, where in fact nearly $60 \%$ of this variation is due to district differences. When the year of study was included in the model at the third level the effect size estimate was 0.183 and was still significant. The variance component estimates did not change much and the year of study was not a significant predictor of the effect sizes and did not explain any of the level-2 or level-3 variance. The estimate of the 
year effect was similar to that in two-level model, small and insignificant. The standard errors of the fixed effects estimates where larger in the three-level model, as expected.

When the third level is not included in the analysis the overwhelming majority of the third level variance is part of the second level variance. Results from previous work on multilevel models have indicated that when the top level is omitted almost all of its variance is captured by the immediate lower level that is present (Moerbeek, 2004). In our example, when the district level was omitted, the between-district variance was captured by the between-study variance.

Insert Table 5 Here

\section{Conclusion}

Multilevel models have been used widely over the last two decades and one of their main advantages is that they take into account the clustering or dependencies in the data. Conceptually, one way to think about clustering is via sampling. If one assumes for example that schools are sampled first, and then classrooms within schools are sampled, a three-level model seems appropriate for analyzing such data. However, if sampling does not take place at different levels fixed effects models seem reasonable. Multilevel methods also seem natural methods for analyzing meta-analytic data and three-level models have advantages over two-level models when there is variability, or clustering, at the third level. In this case, first level-3 units (e.g., school districts) would be sampled first, and then studies within districts would be sampled. Other times however, we are interested empirically in whether clustering takes place at different 
levels because that clustering indicates level-specific effects (e.g., study or district effects). In addition, sometimes, conceptually, we are interested in modeling predictors at the appropriate level. In the calendar data example one could include for instance the evaluator variable as a study-specific predictor or district year or size as a third level predictor. So long as there are enough studies and districts the model should run with no problems.

One advantage of employing three-level models is that such models allow the estimation of variance components of random effects at the third level. For example, a three-level model will estimate the between-researcher variance of study-specific effect size estimates. This model is useful and appropriate when the between level-3 unit variance is different from zero and there are enough level-3 units in the sample to be able to estimate that variance. When the above conditions hold the third level variance should be included in the estimation of regression estimates and their standard errors. If the third level variance is not different from zero however, or when there are only a few level-3 units, one could argue that a two-level model is more appropriate since the level-3 units can be treated as fixed effects (e.g., binary indicators). In the same vein, if the between-study variance in two-level models is not different from zero, or is assumed to be zero by design, then a fixed effects model is appropriate. Nonetheless when variance components are included in the computation of the fixed effects estimates and their standard errors these estimates will be different than in the fixed effects models case. Specifically, the standard errors of the estimates in the random effects models that take into account variance components should be larger than those in the fixed effects models.

Another advantage of the three-level model is that study-specific variables, or slopes, at the second level can be modeled as random effects at the third level (Raudenbush \& Bryk, 2002). Again the assumption is that there are enough level-3 units to compute such variance 
components. The example illustrated earlier was about the evaluator effect that could vary across districts. Now, consider another example that is conceptually a meta-analytic problem. Suppose that the researcher is interested in whether small classes affect the classroom variance in achievement differently than regular classes and whether this effect varies across schools. The classrooms are nested within schools. Each classroom variance (i.e., the effect size) has a known asymptotic variance (i.e., $v_{\mathrm{i}}$ ) (see Raudenbush \& Bryk, 1987). The main independent variable has two categories, small or regular class, is included at the study level, and captures class size effects on the classroom achievement variance. One of the researcher's objectives is whether the class size effects on the classroom achievement variance differ across schools. That is, the class size effects may not be consistent across schools and may interact with school context. In a twolevel model the estimate of the class size effect can’t be treated as random effect. In a three-level model however, the class size estimate can be modeled as a random effect at the third level, the school, and thus the variability of the class size effects across schools can be estimated. In this example, the research question can be addressed by using three-level models that allow the treatment effect to vary across schools.

To conclude, multilevel models are appropriate for modeling meta-analytic data with nested structure and dependencies in the data. Two- or three-level models could be used to model meta-analytic data, and the choice of the model should be supported by the data structure and the sample sizes at each level of the hierarchy as well as the assumptions about the sampling that takes place at each level.

Note:

${ }^{1}$ The $95 \%$ CI for the variance components was constructed using methods by Burdick and Graybill (1992). For example, the 95\% CI for the second level variance component was 
constructed as $v \hat{\tau} / \chi_{v, 1-\alpha / 2}^{2} \leq \tau \leq v \hat{\tau} / \chi_{v, \alpha / 2}^{2}$, where $v=2(\tau / S E(\tau))^{2}$. This formula is used in SAS proc mixed.

\section{Appendix}

The log-likelihood function is

$$
L=-\frac{1}{2} n \log (\pi)-\frac{1}{2} \log (|\mathbf{V}|)-\frac{1}{2} \mathbf{e}^{T} \mathbf{V}^{-1} \mathbf{e},
$$

where $\log$ is the natural logarithm, $\boldsymbol{\pi}$ is a mathematical constant, $\mathbf{V}$ is a $k \times k$ variancecovariance matrix, $\mathbf{e}$ is a $k \times 1$ column vector of residuals, $\mathbf{e}=\mathbf{T}-\mathbf{X} \boldsymbol{\beta}, \mathbf{T}$ is a $k \times 1$ vector of effect sizes, $\mathbf{X}$ is a $k \times k$ matrix of predictors, $\boldsymbol{\beta}$ is a $k \times 1$ vector of fixed effects estimates, and $|\mathbf{V}|$ is the determinant of $\mathbf{V}$.

Two-Level Estimation

In the two-level case $\mathbf{V}_{2}$ is $k \times k$ diagonal variance-covariance matrix with diagonal elements $v_{i}+\tau_{R}$. When no predictors are included at the second level the diagonal elements are $v_{i}+\tau$. The computation of the log-likelihood involves essentially the computation of the inverse and the determinant of matrix $\mathbf{V}_{2}$. The inverse of matrix $\mathbf{V}_{2}$ is also a diagonal matrix with diagonal elements $\left(v_{i}+\tau_{R}\right)^{-1}$.

Similarly, following standard results for determinants of matrices (Harville, 1997) the determinant of the diagonal matrix $\mathbf{V}_{2}$ is the product of the diagonal elements of $\mathbf{V}_{2}$

$$
\left|\mathbf{V}_{2}\right|=\prod_{i=1}^{k}\left(v_{i}+\tau_{R}\right)
$$


and when there are no covariates in the model $\left|\mathbf{V}_{2}\right|=\prod_{i=1}^{k}\left(v_{i}+\tau\right)$. In each iteration the Fisher scoring algorithm updates the fixed effects vector

$$
\widehat{\boldsymbol{\beta}}=\left(\mathbf{X}^{T} \mathbf{V}_{2}^{-1} \mathbf{X}\right)^{-1} \mathbf{X}^{T} \mathbf{V}_{2}^{-1} \mathbf{T}
$$

The asymptotic variance matrix of the fixed effects is computed from the inverse of the expected information matrix, namely

$$
\operatorname{var}(\hat{\boldsymbol{\beta}})=\left(\mathbf{X}^{T} \mathbf{V}_{2}^{-1} \mathbf{X}\right)^{-1}
$$

In the simplest case the computation of the overall mean is updated by

$$
\mathbf{X}^{T} \mathbf{V}_{2}^{-1} \mathbf{X}=\sum_{i=1}^{k}\left(v_{i}+\tau\right)^{-1}, \mathbf{X}^{T} \mathbf{V}_{2}^{-1} \mathbf{T}=\sum_{i=1}^{k} T_{i}\left(v_{i}+\tau\right)^{-1}
$$

The Fisher scoring equation for the variance component when predictors are included in the second level is

$$
\tau_{R}=\tau_{R 0}-2\left\{\sum_{i=1}^{k}\left(v_{i}+\tau_{R}\right)^{-2}\right\}^{-1} \frac{1}{2}\left\{\sum_{i=1}^{k}\left(v_{i}+\tau_{R}\right)^{-1}-\mathbf{e}^{T} \mathbf{V}_{2}^{-2} \mathbf{e}\right\} .
$$

\section{Three-Level Estimation}

When we follow the general formulation for the linear mixed model the three-level metaanalytic model in a single-level equation is

$$
\mathbf{T}=\mathbf{X B}+\mathbf{Z} \boldsymbol{\xi}+\boldsymbol{\varepsilon}
$$

where $\mathbf{T}$ is the vector of effect-size estimates, $\mathbf{X}$ is the design matrix of the fixed effects that includes the level-2 and level-3 predictors, $\mathbf{B}$ is the vector of fixed effects at the second and third levels that need to be estimated, $\mathbf{Z}$ is a design matrix of the random effects at the second 
and third levels, $\boldsymbol{\xi}$ represents the vectors of random effects at the second and third levels, and $\varepsilon$ is the vector of the level-1 sampling errors. The matrix $\mathbf{Z}$ at the second level is $\mathbf{Z}_{(2)}=\mathbf{I}_{k}$, where $\mathbf{I}$ is an identity matrix, and at the third level (assuming no predictors at any level) the matrix $\mathbf{Z}_{(3)}=\mathbf{1}_{k}$ is a vector of ones since the third level random effect involves the level-3 unit intercepts. When level-2 slopes are treated as random effects at the third level the $\mathbf{Z}$ matrix is complicated and includes columns that represent these random slopes.

The variance-covariance matrix $\mathbf{V}_{3}$ is now a $k \times k$ block diagonal matrix

$$
\mathbf{V}_{3}=\mathbf{I}_{m} \otimes\left\{\mathbf{V}_{(3, g)}\right\}=\operatorname{diag}\left\{\mathbf{V}_{\left(3, g_{1}\right)}, \ldots, \mathbf{V}_{\left(3, g_{m}\right)}\right\},
$$

assuming $m$ level-3 units. Each block matrix is

$$
\mathbf{V}_{(3, g)}=\mathbf{V}_{(2, g)}+\mathbf{Z}_{(3, g)} \boldsymbol{\Omega}_{(3) R} \mathbf{Z}_{(3, g)}^{T},
$$

where $\mathbf{V}_{(2, g)}=\mathbf{I}_{n_{g}} \otimes\left\{V_{\left(2, n_{g}\right)}\right\}=\operatorname{diag}\left(v_{1}+\tau_{R}, \ldots, v_{n_{g}}+\tau_{R}\right)$ is a diagonal matrix with elements $v_{i}+\tau_{R}$ and $n_{g}$ is the sample size or the number of studies within a level-3 unit g, and $\otimes$ is the Kronecker product. When no predictors are included at any level the diagonal elements are $v_{i}+\tau$. From standard matrix algebra we know that

$$
\mathbf{V}_{3}=\mathbf{I}_{m} \otimes\left\{\mathbf{V}_{(2, g)}\right\}+\mathbf{I}_{m} \otimes\left\{\mathbf{Z}_{(3, g)} \boldsymbol{\Omega}_{(3) R} \mathbf{Z}_{(3, g)}^{T}\right\},
$$

where $\mathbf{Z}_{(3, g)}$ is the design matrix of the random effects at the third level within level-3 unit g (the subscript $3, g$ indicates unit $g$ at the third level) and $\boldsymbol{\Omega}_{(3) R}$ is the matrix of the third level residual variance components and covariances.

The computation of the log-likelihood involves the computation of the inverse and the determinant of matrix $\mathbf{V}_{3}$. To facilitate these computations I employ standard results from matrix 
algebra (see Harville, 1997; Longford, 1987, 1993). The inverse of matrix $\mathbf{V}_{3}$ is also a block diagonal matrix expressed as

$$
\mathbf{V}_{3}^{-1}=\mathbf{I}_{m} \otimes\left\{\mathbf{V}_{(3, g)}^{-1}\right\}
$$

assuming a total number of $m$ level-3 units where each block is

$$
\mathbf{V}_{(3, g)}^{-1}=\mathbf{V}_{(2, g)}^{-1}-\mathbf{V}_{(2, g)}^{-1} \mathbf{Z}_{(3, g)}\left(\boldsymbol{\Omega}_{(3) R}^{-1}+\mathbf{Z}_{(3, g)}^{T} \mathbf{V}_{(2, g)}^{-1} \mathbf{Z}_{(3, g)}\right)^{-1} \mathbf{Z}_{(3, g)}^{T} \mathbf{V}_{(2, g)}^{-1},
$$

and

$$
\mathbf{V}_{(2, g)}^{-1}=\mathbf{I}_{n_{g}} \otimes\left\{V_{\left(2, n_{g}\right)}^{-1}\right\}, \quad V_{\left(2, n_{g}\right)}^{-1}=\left(v_{i}+\tau_{R}\right)^{-1},
$$

that is, $\mathbf{V}_{(2, g)}^{-1}$ is a diagonal matrix with elements $\left(v_{i}+\tau_{R}\right)^{-1}$.

Similarly, following standard results for determinants of matrices (Harville, 1997) the determinant of the block-diagonal matrix $\mathbf{V}_{3}$ is

$$
\left|\mathbf{V}_{3}\right|=\prod_{g=1}^{m}\left|\mathbf{V}_{(3, g)}\right|
$$

and the determinant of $\mathbf{V}_{(3, g)}$ is

$$
\left|\mathbf{V}_{(3, g)}\right|=\left|\mathbf{V}_{(2, g)}\left\|\boldsymbol{\Omega}_{(3) R}\right\| \boldsymbol{\Omega}_{(3) R}^{-1}+\mathbf{Z}_{(3, g)}^{T} \mathbf{V}_{(2, g)}^{-1} \mathbf{Z}_{(3, g)}\right|
$$

where

$$
\left|\mathbf{V}_{(2, g)}\right|=\prod_{i=1}^{n_{g}} V_{\left(2, n_{g}\right)}=\prod_{i=1}^{n_{g}}\left(v_{i}+\tau_{R}\right)
$$

The first- and second-order derivatives of the log-likelihood equation with respect to the second level variance component $\tau$ are

$$
-\frac{1}{2}\left\{\sum_{g=1}^{m} \operatorname{tr}\left\{\mathbf{V}_{(3, g)}^{-1}\right\}-\sum_{g=1}^{m}\left\{\left(\mathbf{e}_{(3, g)}^{T} \mathbf{V}_{(3, g)}^{-1}\right)\left(\mathbf{V}_{(3, g)}^{-1} \mathbf{e}_{(3, g)}\right)\right\}_{g}\right\},
$$


and

$$
\frac{1}{2} \sum_{g=1}^{m} \operatorname{tr}\left\{\mathbf{V}_{(3, g)}^{-1} \mathbf{V}_{(3, g)}^{-1}\right\}_{g}
$$

respectively, where $\operatorname{tr}(\mathbf{A})$ is the trace of matrix $\mathbf{A}$, the sum of diagonal elements of $\mathbf{A}$. The derivative $\frac{\partial \mathbf{V}}{\partial \tau}$ produces an identity matrix that is not used in the computation.

The first-order derivatives of the log-likelihood equation of the third level variance component matrix $\boldsymbol{\Omega}_{(3) R}$ for each variance or unique covariance is

$$
-\frac{1}{2}\left\{\sum_{g=1}^{m}\left\{\left(\mathbf{Z}_{(3, g)}^{T} \mathbf{V}_{(3, g)}^{-1} \mathbf{Z}_{(3, g)}\right)_{j l}\right\}_{g}-\sum_{g=1}^{m}\left\{\left(\mathbf{Z}_{(3, g)}^{T} \mathbf{V}_{(3, g)}^{-1} \mathbf{e}_{(3, g)}\right)_{j}\left(\mathbf{Z}_{(3, g)}^{T} \mathbf{V}_{(3, g)}^{-1} \mathbf{e}_{(3, g)}\right)_{l}\right\}_{g}\right\}
$$

where $j, l$ indicate the $j$ th row and lth column element of the matrix. The derivative $\frac{\partial \Omega_{(3) R}}{\partial \omega_{R j l}}$ produces an incidence matrix with zeros everywhere and one for element $j, l$ (Longford, 1993).

The diagonal elements (variances) of the expected information matrix for the third-level variance components (i.e., the variances and lower triangular matrix covariances) are computed as

$$
\frac{1}{2} \sum_{g=1}^{m}\left\{\left(\mathbf{Z}_{(3, g)}^{T} \mathbf{V}_{(3, g)}^{-1} \mathbf{Z}_{(3, g)}\right)_{j l}\left(\mathbf{Z}_{(3, g)}^{T} \mathbf{V}_{(3, g)}^{-1} \mathbf{Z}_{(3, g)}\right)_{j l}\right\}_{g}
$$

To compute the off-diagonal elements (covariances) between the variance and the covariance components we use

$$
\sum_{g=1}^{m}\left\{\left(\mathbf{Z}_{(3, g)}^{T} \mathbf{V}_{(3, g)}^{-1} \mathbf{Z}_{(3, g)}\right)_{j m}\left(\mathbf{Z}_{(3, g)}^{T} \mathbf{V}_{(3, g)}^{-1} \mathbf{Z}_{(3, g)}\right)_{k l}\right\}_{g}
$$


Two-level unconditional meta-analysis using proc mixed in SAS

proc mixed data=temp covtest;

class studyid;

model effectsize $=/$ solution $\operatorname{ddfm}=$ bw notest ;

random int / sub = studyid;

repeated / group = studyid;

parms $(0.1)$

$(0.118)(0.118)(0.144)(0.144)(0.014)(0.014)(0.015)(0.024)(0.023)(0.043)$

$(0.012)(0.020)(0.004)(0.004)(0.007)(0.019)(0.007)(0.005)(0.004)(0.020)$

$(0.018)(0.019)(0.022)(0.020)(0.021)(0.006)(0.007)(0.007)(0.007)(0.007)$

$(0.015)(0.011)(0.010)(0.001)(0.001)$ ( 0.001$)$ ( 0.001$)(0.001)$ ( 0.001$)(0.001)$

$(0.001)(0.010)(0.011)(0.010)(0.009)(0.013)(0.013)(0.031)(0.031)(0.030)$

$(0.030)(0.030)(0.087)(0.082)(0.067)(0.067)$

/ eqcons $=2$ to 57 ;

run;

Three-level unconditional meta-analysis using proc mixed in SAS

proc mixed data=temp covtest;

class districtid studyid;

model effectsize $=/$ solution ddfm $=$ bw notest ;

random int / sub = districtid;

random int / sub = studyid(districtid);

repeated $/$ group $=$ studyid $($ districtid $)$;

parms $(0.1)(0.1)$

$(0.118)(0.118)(0.144)(0.144)(0.014)(0.014)(0.015)(0.024)(0.023)(0.043)$

$(0.012)(0.020)(0.004)(0.004)(0.007)(0.019)(0.007)(0.005)(0.004)(0.020)$

$(0.018)(0.019)(0.022)(0.020)(0.021)(0.006)$ ( 0.007$)(0.007)(0.007)(0.007)$

$(0.015)(0.011)(0.010)(0.001)(0.001)(0.001)(0.001)(0.001)(0.001)(0.001)$

$(0.001)(0.010)(0.011)(0.010)(0.009)(0.013)(0.013)(0.031)(0.031)(0.030)$

$(0.030)(0.030)(0.087)(0.082)(0.067)(0.067)$

/ eqcons $=3$ to 58 ;

run; 
Two-level unconditional meta-analysis using HLM

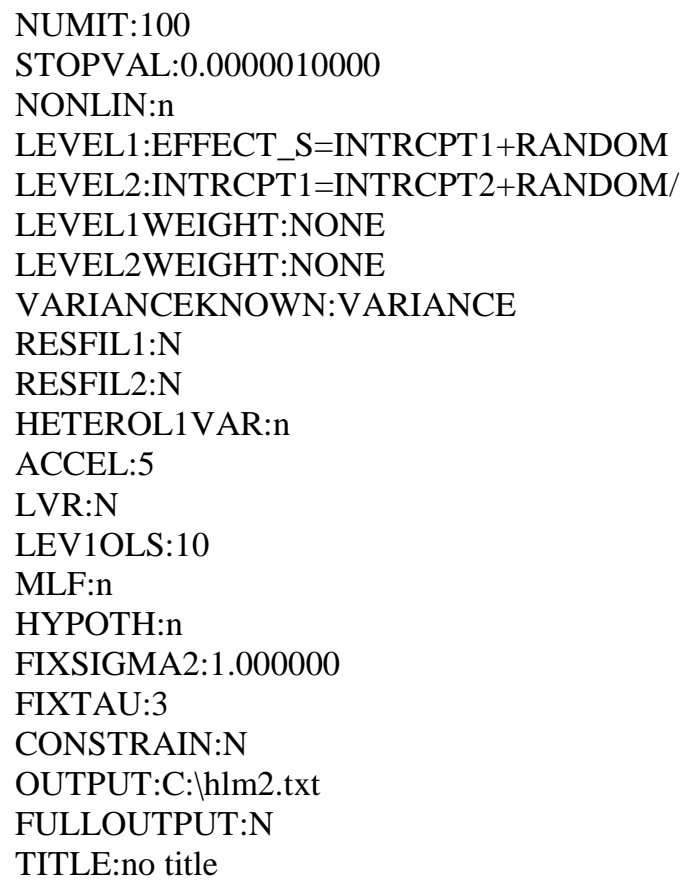

Three-level unconditional meta-analysis using HLM

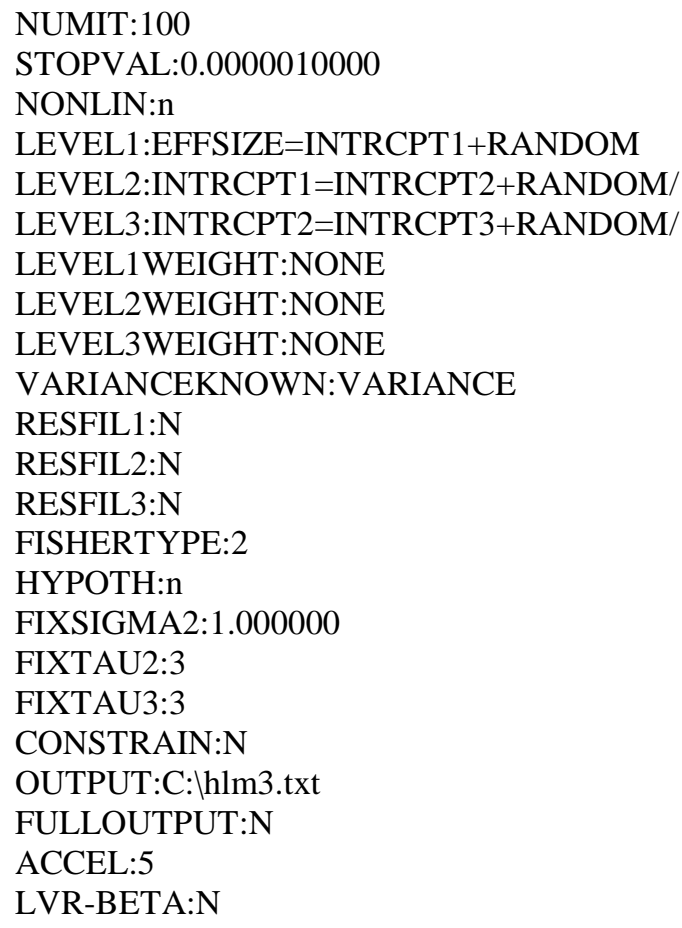




\section{References}

Berkey, C. S., Anderson, J. J., \& Hoaglin, D. C. (1996). Multiple-outcome meta-analysis of clinical trials. Statistics in Medicine, 15, 537-557.

Bryk, A. S., \& Raudenbush, S. W. (1988). Toward a more appropriate conceptualization of research on school effects. A three-level hierarchical linear model. American Journal of Education, 97, 65-108.

Burdick, R. K., \& Graybill, F. A. (1992). Confidence intervals on variance components. New York: Marcel Dekker.

Cooper, H., Hedges, L. V., \& Valentine, J. C. (2009). The handbook of research synthesis and meta-analysis ( $\left.2^{\text {nd }} \mathrm{Ed}\right)$. New York: Russell Sage.

Cooper, H., Valentine, J. C., Charlton, K., \& Melson, A. (2003). The effects of modified school calendars on student achievement and on school and community attitudes: A research synthesis. Review of Educational Research, 73, 1-52.

DerSimonian, R., \& Laird, N. (1986). Meta-analysis in clinical trials. Controlled Clinical Trials, 7, 177-188.

Garvey, W. \& Griffith, B. (1971). Scientific communication: Its role in the conduct of research and creation of knowledge. American Psychologist, 26, 349-361.

Glass, G. V. (1976). Primary, secondary, and meta-analysis. Educational Researcher,5, 3 $-8$.

Gleser, L. J., \& Olkin, I. (2009). Stochastically dependent effect sizes. In H. Cooper, L. V. Hedges, \& J. C. Valentine The handbook of research synthesis and meta-analysis (pp. 357-376). New York: Russell Sage.

Goldstein, H. (1987). Multilevel models in educational and social research. London: Oxford University Press.

Goldstein, H., Yang, M., Omar, R., Turner, R., \& Thompson, S. (2000). Meta-analysis using multilevel models with an application to the study of class size effects. Journal of the Royal Statistical Society Series C-Applied Statistics, 49, 399-412.

Harville, D. A. (1997). Matrix algebra from a statistician's perspective. New York: Springer.

Hedges, L. V. (1982). Estimation of effect size from a series of independent experiments. Psychological Bulletin, 92, 490-499. 
Hedges, L. V. (1983). A random effects model for effect sizes. Psychological Bulletin, 93, 388395.

Hedges, L. V., \& Olkin, I. (1985). Statistical methods for meta-analysis. Orlando, FL: Academic Press.

Hedges, L. V., \& Vevea, J. L. (1998). Fixed and random effects models in meta analysis. Psychological Methods, 3, 486-504.

Hox, J. J., \& de Leeuw, E. D. (2003). Multilevel models for meta-analysis. In S.P. Reise \& N. Duan (Eds.) Multilevel Modeling: Methodological Advances, Issues, and Applications (pp. 90- 111). Mahwah, NJ: Lawrence Erlbaum Associates.

Konstantopoulos, S. (2003). Variance components estimation in three-level meta-analysis. Unpublished doctoral dissertation.

Konstantopoulos, S. (2007). Introduction to meta-analysis. In J. Osbourne (Ed.), Best practices in quantitative methods (pp. 177-194). Thousand Oaks, CA: Sage.

Konstantopoulos, S., \& Hedges, L.V. (2004). Meta-Analysis. In D. Kaplan (Ed.), Handbook of Quantitative Methodology for the Social Sciences (pp. 281-297). New York: Sage.

Lipsey, M. W., \& Wilson, D. B. (2001). Practical meta-analysis. Thousand Oaks, CA: Sage.

Littell, R. C., Milliken, G. A., Stroup, W. W., \& Wolfinger, R. D. (1996). SAS system for mixed models. Cary, NC: SAS Institute INC.

Longford, N T. (1987). A fast scoring algorithm for maximum likelihood estimation in unbalanced mixed models with nested random effects. Biometrika, 74, 817-827.

Longford, N T. (1993). Random coefficient models. New York: Oxford University Press.

Moerbeek, M. (2004). The consequences of ignoring a level of nesting in multilevel analysis. Multivariate Behavioral Research, 39, 129-149.

Nye, B., Konstantopoulos, S, \& Hedges, L.V. (2004). How Large are Teacher Effects? Educational Evaluation and Policy Analysis, 26, 237-257.

Raudenbush, S. W., \& Bryk, A. S. (1987). Examining correlates of diversity. Journal of Educational Statistics, 12, 241-269.

Raudenbush, S. W., \& Bryk, A. S. (2002). Hierarchical linear models. Newbury Park, CA: Sage. 
Raudenbush, S. W., Bryk, A., Cheong, Y. F., \& Congdon, R. (2004). HLM 6:

Hierarchical linear and onlinear modeling. Lincolnwood, IL: Scientific Software International.

Singer, J. D. (1998). Using SAS PROC MIXED to fit multilevel growth models, hierarchical models, and individual growth models. Journal of Educational and Behavioral Statistics, 24, 323-355.

Snijders, T. A. B., \& Bosker, R. J. (1999). Multilevel analysis. London: Sage.

Stevens, J. R., \& Taylor, A. M. (2009). Hierarchical dependence in meta-analysis. Journal of Educational and Behavioral Statistics, 34, 46-73. 
Table 1. Data Used in the Analysis

\begin{tabular}{|c|c|c|c|c|}
\hline District ID & Study ID & Effect Size & Variance & Year \\
\hline 11 & 1 & -0.18 & 0.118 & 1976 \\
\hline 11 & 2 & -0.22 & 0.118 & 1976 \\
\hline 11 & 3 & 0.23 & 0.144 & 1976 \\
\hline 11 & 4 & -0.30 & 0.144 & 1976 \\
\hline 12 & 5 & 0.13 & 0.014 & 1989 \\
\hline 12 & 6 & -0.26 & 0.014 & 1989 \\
\hline 12 & 7 & 0.19 & 0.015 & 1989 \\
\hline 12 & 8 & 0.32 & 0.024 & 1989 \\
\hline 18 & 9 & 0.45 & 0.023 & 1994 \\
\hline 18 & 10 & 0.38 & 0.043 & 1994 \\
\hline 18 & 11 & 0.29 & 0.012 & 1994 \\
\hline 27 & 12 & 0.16 & 0.020 & 1976 \\
\hline 27 & 13 & 0.65 & 0.004 & 1976 \\
\hline 27 & 14 & 0.36 & 0.004 & 1976 \\
\hline 27 & 15 & 0.60 & 0.007 & 1976 \\
\hline 56 & 16 & 0.08 & 0.019 & 1997 \\
\hline 56 & 17 & 0.04 & 0.007 & 1997 \\
\hline 56 & 18 & 0.19 & 0.005 & 1997 \\
\hline 56 & 19 & -0.06 & 0.004 & 1997 \\
\hline 58 & 20 & -0.18 & 0.020 & 1976 \\
\hline 58 & 21 & 0.00 & 0.018 & 1976 \\
\hline 58 & 22 & 0.00 & 0.019 & 1976 \\
\hline 58 & 23 & -0.28 & 0.022 & 1976 \\
\hline 58 & 24 & -0.04 & 0.020 & 1976 \\
\hline 58 & 25 & -0.30 & 0.021 & 1976 \\
\hline 58 & 26 & 0.07 & 0.006 & 1976 \\
\hline 58 & 27 & 0.00 & 0.007 & 1976 \\
\hline 58 & 28 & 0.05 & 0.007 & 1976 \\
\hline 58 & 29 & -0.08 & 0.007 & 1976 \\
\hline 58 & 30 & -0.09 & 0.007 & 1976 \\
\hline 71 & 31 & 0.30 & 0.015 & 1997 \\
\hline 71 & 32 & 0.98 & 0.011 & 1997 \\
\hline 71 & 33 & 1.19 & 0.010 & 1997 \\
\hline 86 & 34 & -0.07 & 0.001 & 1997 \\
\hline 86 & 35 & -0.05 & 0.001 & 1997 \\
\hline 86 & 36 & -0.01 & 0.001 & 1997 \\
\hline 86 & 37 & 0.02 & 0.001 & 1997 \\
\hline 86 & 38 & -0.03 & 0.001 & 1997 \\
\hline 86 & 39 & 0.00 & 0.001 & 1997 \\
\hline 86 & 40 & 0.01 & 0.001 & 1997 \\
\hline 86 & 41 & -0.10 & 0.001 & 1997 \\
\hline 91 & 42 & 0.50 & 0.010 & 2000 \\
\hline 91 & 43 & 0.66 & 0.011 & 2000 \\
\hline 91 & 44 & 0.20 & 0.010 & 2000 \\
\hline 91 & 45 & 0.00 & 0.009 & 2000 \\
\hline 91 & 46 & 0.05 & 0.013 & 2000 \\
\hline 91 & 47 & 0.07 & 0.013 & 2000 \\
\hline 108 & 48 & -0.52 & 0.031 & 2000 \\
\hline 108 & 49 & 0.70 & 0.031 & 2000 \\
\hline 108 & 50 & -0.03 & 0.030 & 2000 \\
\hline 108 & 51 & 0.27 & 0.030 & 2000 \\
\hline 108 & 52 & -0.34 & 0.030 & 2000 \\
\hline 644 & 53 & 0.12 & 0.087 & 1995 \\
\hline 644 & 54 & 0.61 & 0.082 & 1995 \\
\hline 644 & 55 & 0.04 & 0.067 & 1994 \\
\hline 644 & 56 & -0.05 & 0.067 & 1994 \\
\hline
\end{tabular}


Table 2. Summary Statistics

\begin{tabular}{lcccc}
\hline & \multicolumn{4}{c}{ Reading } \\
\cline { 2 - 5 } & Mean & SD & Min & Max \\
\hline Effect Size Estimate & 0.120 & 0.326 & -0.52 & 1.19 \\
Type of Calendar & $51.79 \%$ & 0.504 & 0.00 & 1.00 \\
Sample Size Across Studies & 913.018 & 1459.680 & 28.00 & 4403.00 \\
\hline
\end{tabular}


Table 3. Descriptive Statistics by District

\begin{tabular}{ccccc}
\hline & \multicolumn{4}{c}{ Reading } \\
\cline { 2 - 5 } District ID & Mean & SD & Min & Max \\
\hline 11 & -0.118 & 0.237 & -0.30 & 0.23 \\
12 & 0.095 & 0.250 & -0.26 & 0.32 \\
18 & 0.373 & 0.080 & 0.29 & 0.45 \\
27 & 0.443 & 0.227 & 0.16 & 0.60 \\
56 & 0.061 & 0.103 & -0.06 & 0.19 \\
58 & -0.077 & 0.126 & -0.28 & 0.07 \\
71 & 0.823 & 0.465 & 0.30 & 1.19 \\
86 & -0.029 & 0.042 & -0.10 & 0.02 \\
91 & 0.247 & 0.271 & 0.00 & 0.66 \\
108 & 0.016 & 0.487 & -0.52 & 0.70 \\
644 & 0.180 & 0.295 & -0.05 & 0.61 \\
\hline
\end{tabular}


Table 4. Two-Level Estimates of Fixed Effects and Variance Components

\begin{tabular}{lcccccc}
\hline & \multicolumn{3}{c}{ Unconditional Model } & \multicolumn{3}{c}{ Including Year of Study } \\
\cline { 2 - 7 } & Estimates & SE & $95 \%$ CI & Estimates & SE & 95\% CI \\
\hline Fixed Effects & & & & & & \\
$\quad$ Intercept & $0.128^{*}$ & 0.044 & $(0.040,0.216)$ & $0.126^{*}$ & 0.043 & $(0.040,0.212)$ \\
$\quad$ Year of Study & - & - & - & 0.005 & 0.004 & $(-0.003,0.013)$ \\
& & & & & & \\
Variance Components & & & & & & \\
$\quad$ Second Level & $0.088^{*}$ & 0.020 & $(0.059,0.146)$ & $0.088^{*}$ & 0.020 & $(0.059,0.146)$ \\
\hline$* \mathrm{p}<0.05$
\end{tabular}


Table 5. Three-Level Estimates of Fixed Effects and Variance Components

\begin{tabular}{lccccccc}
\hline & \multicolumn{3}{c}{ Unconditional Model } & \multicolumn{3}{c}{ Including Year of Study } \\
\cline { 2 - 8 } & Estimates & SE & $95 \%$ CI & Estimates & SE & 95\% CI \\
\hline Fixed Effects & & & & & & & \\
$\quad$ Intercept & $0.184^{*}$ & 0.080 & $(0.006,0.362)$ & $0.183^{*}$ & 0.080 & $(0.002,0.364)$ \\
$\quad$ Year of Study & - & - & - & 0.005 & 0.009 & $(-0.015,0.025)$ \\
& & & & & & & \\
Variance Components & & & & & & & \\
$\quad$ Second Level & $0.033^{*}$ & 0.010 & $(0.020,0.070)$ & $0.033^{*}$ & 0.010 & $(0.020,0.070)$ \\
$\quad$ Third Level & $0.058^{*}$ & 0.030 & $(0.027,0.256)$ & $0.056^{*}$ & 0.030 & $(0.027,0.315)$ \\
\hline $\mathrm{p}<0.05$ & & & & & & &
\end{tabular}

